\title{
ON THE ASYMPTOTIC JOINT DISTRIBUTION OF HEIGHT AND WIDTH IN RANDOM TREES
}

\author{
SVANTE JANSON \\ Department of Mathematics, Uppsala University, PO Box 480, S-751 06 Uppsala, Sweden \\ e-mail: svante.janson@math.uu.se \\ Communicated by E. Csáki
}

(Received February 2, 2006; accepted April 18, 2006)

\begin{abstract}
It has been known for a long time that the height and width of a random labelled rooted tree, or of any other conditioned Galton-Watson tree, after suitable normalizations converge to the same limit distribution. Moreover, Chassaing, Marckert and Yor [7] have proved joint convergence of height and width. The resulting two-dimensional limit distribution has been studied by Donati-Martin [10]. We extend her results and give new formulas for joint moments. As an example, we calculate the covariance. We also show that the two-dimensional distribution is not symmetric, although the marginals are the same.
\end{abstract}

\section{Introduction}

If $T$ is a rooted tree, the depth $d(v)$ of a vertex $v$ is the distance from $v$ to the root, the profile of $T$ is the sequence $w_{k}:=\#\{v: d(v)=k\}$, $k=0,1, \ldots$, of sizes of the levels in $T$, the height $h(T)$ of $T$ is the maximal depth, i.e. $h(T):=\max _{v} d(v)=\max \left\{k: w_{k}>0\right\}$, and the width $w(T)$ of $T$ is the maximal size of a level in $T$, i.e. $w(T):=\max _{k} w_{k}$.

Let $T_{n}$ be a random conditioned Galton-Watson tree with $n$ vertices, i.e. a random tree obtained as the family tree of a Galton-Watson process conditioned on a given total population of $n$. (See e.g. [1,9] for details.) The Galton-Watson process is defined using an offspring distribution; let

2000 Mathematics Subject Classification. Primary 60J65; Secondary 60 C05.

Key words and phrases. Conditioned Galton-Watson trees, random trees, height, width, Brownian excursion. 
$\xi$ denotes a random variable with this distribution. We assume, as usual, $\mathbb{E} \xi=1$ (the Galton-Watson process is critical) and $0<\sigma^{2}=\operatorname{Var} \xi<\infty$.

It is well-known [1] that the conditioned Galton-Watson trees are the same as the simply generated trees [19]. Many combinatorially interesting random trees are of this type, with different choices of $\xi$, for example labelled trees $\left(\xi \sim \operatorname{Po}(1), \sigma^{2}=1\right)$; ordered (=plane) trees $(\mathbb{P}(\xi=k)=$ $\left.2^{-k-1}, \sigma^{2}=2\right)$; binary trees $\left(\xi \sim \operatorname{Bi}(2,1 / 2), \sigma^{2}=1 / 2\right)$; strict binary trees $\left(\mathbb{P}(\xi=0)=\mathbb{P}(\xi=2)=1 / 2, \sigma^{2}=1\right)$.

It is also well-known that many asymptotic properties are the same for different conditioned Galton-Watson trees, except that the offspring variance $\sigma^{2}$ appears as a scale parameter; this is explained by Aldous' theory of the continuum random tree $[1,2]$. In particular, there exist random variables $H$ and $W$ such that for any conditioned Galton-Watson tree $T_{n}$

$$
n^{-1 / 2}\left(h\left(\mathcal{T}_{n}\right), w\left(\mathcal{T}_{n}\right)\right) \stackrel{\mathrm{d}}{\longrightarrow}\left(\sigma^{-1} H, \sigma W\right) .
$$

The joint convergence (1.1) was first given by Chassaing, Marckert and Yor [7], see also [15]. The separate convergences $n^{-1 / 2} h\left(\mathcal{T}_{n}\right) \stackrel{\mathrm{d}}{\longrightarrow} \sigma^{-1} H$ and $n^{-1 / 2} w\left(\mathcal{T}_{n}\right) \stackrel{\mathrm{d}}{\longrightarrow} \sigma W$ have been known for a long time, see $[1,2,3,6,11,12$, $13,21,23]$.

The joint distribution of $H$ and $W$ can be expressed using a normalized Brownian excursion $B_{\text {ex }}$ in (at least) two different ways, both explained in [7] as consequences of two different proofs of (1.1).

First, using the depth-first walk of the tree, one obtains (1.1) with

$$
(H, W) \stackrel{\mathrm{d}}{=}\left(2 \max _{t} B_{\mathrm{ex}}(t), \frac{1}{2} \max _{x \geqq 0} \ell(x)\right)
$$

where $\ell$ is the local time of $B_{\text {ex }}$, see $[1,3,11]$.

Secondly, using instead the breadth-first walk, one obtains (1.1) with

$$
(H, W) \stackrel{\mathrm{d}}{=}\left(\int_{0}^{1} \frac{d t}{B_{\mathrm{ex}}(t)}, \max _{t} B_{\mathrm{ex}}(t)\right),
$$

see $[7,15]$. Of course, the right hand sides of (1.2) and (1.3) are not the same, but we see that they are equal in distribution. The relation between them can be explained by Jeulin's description [16] of $\frac{1}{2} \ell(x)$ as a time change of another Brownian excursion, see [7]. 
In particular, (1.2) and (1.3) imply the identity by Biane and Yor [5]

$$
2 \max _{t} B_{\mathrm{ex}}(t) \stackrel{\mathrm{d}}{=} \int_{0}^{1} \frac{d t}{B_{\mathrm{ex}}(t)}
$$

which by (1.3) yields the well-known

$$
H \stackrel{\mathrm{d}}{=} 2 W .
$$

$H$ and $W$ thus have the same distribution, except for a scale factor. This distribution has been much studied, see for example $[8,17,18,21]$ and, with many connections to other random variables and functions, [4]. In particular, it is known that

$$
\mathbb{P}(W \leqq x)=\sum_{k=-\infty}^{\infty}\left(1-4 k^{2} x^{2}\right) e^{-2 k^{2} x^{2}}, \quad x>0
$$

further, the moment $\mathbb{E} W^{s}$ is finite for every real (positive or negative) $s$ and, for any complex $s$,

$$
\mathbb{E} W^{s}=2^{-s / 2} s(s-1) \Gamma(s / 2) \zeta(s)=2(\pi / 2)^{s / 2} \xi(s),
$$

where $\zeta$ is the Riemann zeta function, and $\xi$ is the related Riemann xi function.

The joint distribution of $H$ and $W$ has been studied by Donati-Martin [10]. She used the agreement formula by Pitman and Yor [20, 24] and derived various formulas, including the formula (2.1) below for mixed moments $\mathbb{E}\left(W^{s} H^{t}\right)$ of $H$ and $W$ when $\operatorname{Re}(s+t)>1$ and $\operatorname{Re}(t)<0$. Note that, as remarked above, $W$ has finite moments of all orders, both positive and negative, and the same holds for $H$ too by (1.5); hence, the Cauchy-Schwarz inequality implies that the mixed moment $\mathbb{E}\left(W^{s} H^{t}\right)$ exists for every complex $s$ and $t$, and that it is an entire function of $(s, t) \in \mathbb{C}^{2}$.

The purpose of this paper is to use Donati-Martin's results and analytic continuation to obtain formulas for a wider range of $s$ and $t$, in particular for positive integers. For example, we calculate the covariance of $H$ and $W$; as might be expected, this is negative. Another simple consequence is following result:

Theorem 1.1. The distribution of $(H, 2 W)$ has two identical marginals, but is not symmetric: $(H, 2 W) \stackrel{\mathrm{d}}{\neq}(2 W, H)$. 
REMARK 1.2. Recall that the Brownian excursion equals a Bessel bridge of index $1 / 2$ (dimension 3). Donati-Martin [10, (5.1)] actually treats the case of a pair of variables defined as in (1.3) from a Bessel bridge of arbitrary index $\nu>-1$. Presumably, the results below extend to this case in a straightforward way, but we have not pursued this.

Acknowledgement. I thank Philippe Chassaing for interesting discussions.

\section{Results}

Let $\Phi(\alpha, \gamma ; z)$ denote the confluent hypergeometric function (sometimes denoted $\left.{ }_{1} F_{1}(\alpha ; \gamma ; z)\right)$, defined for $\gamma \neq 0,-1,-2, \ldots$ and $z \in \mathbb{C}$ by

$$
\Phi(\alpha, \gamma ; z):=\sum_{n=0}^{\infty} \frac{\alpha^{\bar{n}} z^{n}}{\gamma^{\bar{n}} n !}
$$

where $\lambda^{\bar{n}}=\lambda(\lambda+1) \ldots(\lambda+n-1)$ is the rising factorial power. Further, let $A_{\nu}(\lambda, \alpha)=\exp (\lambda) / \Phi\left(\frac{\alpha^{2}}{2 \lambda}+\nu+1 / 2,1+2 \nu ; 2 \lambda\right)$. Then Donati-Martin's formula [10, Corollary 5.1] is, for $\operatorname{Re}(t)<0$ and $\operatorname{Re}(s+t)>1$ (which implies $\operatorname{Re}(s)>1)$ :

$$
\begin{aligned}
\mathbb{E}\left(W^{s} H^{t}\right) & =\sqrt{\frac{\pi}{2}} \frac{2^{(5+t-s) / 2}}{\Gamma(-t) \Gamma\left(\frac{s+t-1}{2}\right)} \int_{0}^{\infty} \int_{0}^{\infty} \lambda^{s+t-2} \alpha^{-(1+2 t)} A_{1 / 2}^{2}(\lambda, \alpha) d \alpha d \lambda \\
& =\frac{2^{2+t / 2-s / 2} \pi^{1 / 2}}{\Gamma(-t) \Gamma\left(\frac{s+t-1}{2}\right)} \int_{0}^{\infty} \int_{0}^{\infty} \frac{\lambda^{s+t-2} \alpha^{-1-2 t} e^{2 \lambda} d \alpha d \lambda}{\Phi^{2}\left(1+\alpha^{2} /(2 \lambda), 2,2 \lambda\right)}
\end{aligned}
$$

We define

$$
F(x, y):=\sum_{n=0}^{\infty} \frac{(x+y)(2 x+y) \cdots(n x+y)}{n !(n+1) !}
$$

Then $F$ is an entire function in $\mathbb{C}^{2}$, and for $x \neq 0$,

$$
F(x, y)=\sum_{n=0}^{\infty} \frac{(1+y / x)^{\bar{n}} x^{n}}{2^{\bar{n}} n !}=\Phi(1+y / x, 2 ; x) .
$$

We can extend Donati-Martin's formula (2.1) as follows, where $\mathrm{D}_{y}$ denotes the partial derivative $\partial / \partial y$. 
TheOREm 2.1. For any complex numbers $s$ and $t$ with $\operatorname{Re}(s+t)>1$, and any integer $m \geqq 0$ with $m>\operatorname{Re}(t)$,

(2.4) $\mathbb{E}\left(W^{s} H^{t}\right)$

$$
=(-1)^{m} \frac{2^{2-t / 2-3 s / 2} \pi^{1 / 2}}{\Gamma(m-t) \Gamma\left(\frac{s+t-1}{2}\right)} \int_{0}^{\infty} \int_{0}^{\infty} x^{s+t-2} y^{m-1-t} e^{x} \mathrm{D}_{y}^{m}\left(F(x, y)^{-2}\right) d x d y .
$$

For integers $t \geqq 0$ we also obtain a simpler formula.

Theorem 2.2. For any integer $k \geqq 0$ and any complex $s$ with $\operatorname{Re}(s)+$ $k>1$,

$$
\mathbb{E}\left(W^{s} H^{k}\right)=(-1)^{k} \frac{2^{2-k / 2-3 s / 2} \pi^{1 / 2}}{\Gamma\left(\frac{s+k-1}{2}\right)} \int_{0}^{\infty} x^{s+k-2} e^{x} \psi_{k}(x) d x
$$

where $\psi_{k}(x):=\left.\mathrm{D}_{y}^{k}\left(F(x, y)^{-2}\right)\right|_{y=0}$.

Define

$$
f_{k}(x):=\left.\mathrm{D}_{y}^{k} F(x, y)\right|_{y=0}, \quad k=0,1,2, \ldots
$$

The functions $\psi_{k}$ can be expressed in $f_{0}, f_{1}, \ldots, f_{k}$ by successive differentiations. In the special cases $k=1$ and $k=2$ we obtain the following. (Similar but more complicated formulas may be given for higher $k$, too.)

Corollary 2.3. For any $s$ with $\operatorname{Re}(s)>0$,

$$
\mathbb{E}\left(W^{s} H\right)=\frac{2^{5 / 2-3 s / 2} \pi^{1 / 2}}{\Gamma(s / 2)} \int_{0}^{\infty} x^{s+2} \frac{e^{x}}{\left(e^{x}-1\right)^{3}} f_{1}(x) d x
$$

Corollary 2.4. For any $s$ with $\operatorname{Re}(s)>-1$,

$$
\begin{aligned}
\mathbb{E}\left(W^{s} H^{2}\right)=\frac{2^{2-3 s / 2} \pi^{1 / 2}}{\Gamma((s+1) / 2)}\left(3 \int_{0}^{\infty} x^{s+4} \frac{e^{x}}{\left(e^{x}-1\right)^{4}} f_{1}(x)^{2} d x\right. \\
\left.\quad-\int_{0}^{\infty} x^{s+3} \frac{e^{x}}{\left(e^{x}-1\right)^{3}} f_{2}(x) d x\right) .
\end{aligned}
$$


We can evaluate the integrals in Corollaries 2.3 and 2.4 as infinite sums, leading to the following, where we use the notation $H_{n}:=\sum_{1}^{n} k^{-1}$ and $H_{n}^{(2)}:=\sum_{1}^{n} k^{-2}$ for the harmonic and second order harmonic numbers [14]; we also write $z !:=\Gamma(z+1)$ for any complex $z$. The sums converge geometrically and can be used for numerical evaluation, see the examples below.

Theorem 2.5. For any $s \neq 0,-1,-2, \ldots$,

$$
\mathbb{E}\left(W^{s} H\right)=\frac{2^{3 / 2-3 s / 2} \pi^{1 / 2}}{\Gamma(s / 2)} \sum_{n=1}^{\infty} H_{n} \frac{(n+s+1) !}{(n+1) !}(\zeta(n+s)-\zeta(n+s+1)) .
$$

TheOREM 2.6. For any $s \neq-1,-2, \ldots$,

$$
\begin{aligned}
& \mathbb{E}\left(W^{s} H^{2}\right) \\
= & \frac{2^{1-3 s / 2} \pi^{1 / 2}}{\Gamma((s+1) / 2)}\left(\sum_{m=0}^{\infty} \sum_{n=0}^{\infty} H_{m+1} H_{n+1} \frac{(m+n+s+4) !}{(m+2) !(n+2) !}\right. \\
& \times(\zeta(m+n+s+2)-3 \zeta(m+n+s+3)+2 \zeta(m+n+s+4)) \\
& \left.-\sum_{n=0}^{\infty}\left(H_{n+2}^{2}-H_{n+2}^{(2)}\right) \frac{(n+s+3) !}{(n+3) !}(\zeta(n+s+2)-\zeta(n+s+3))\right) .
\end{aligned}
$$

When $s$ is a negative integer, the value can be obtained from these formulas as a limit. In particular, we have the following finite sum.

TheOREm 2.7. For any integer $m \geqq 0$,

$$
\begin{aligned}
\mathbb{E}\left(W^{-2 m} H\right)=(-1)^{m} 2^{3 m+1 / 2} \pi^{1 / 2} m ! & \\
\times\left(\sum_{n=1}^{2 m-2} \frac{(-1)^{n} H_{n}}{(2 m-2-n) !(n+1) !}(\zeta(n-2 m)-\zeta(n+1-2 m))\right. & \\
& \left.+2 \frac{H_{2 m+1}}{(2 m+2) !}-\frac{H_{2 m}}{(2 m+1) !}\right) .
\end{aligned}
$$

More generally, but less explicitly, we have the following. Note that this too yields a closed form (a rational number times $\sqrt{2 \pi}$ ) for every $k$ and $m$.

Theorem 2.8. For any integers $k, m \geqq 0$,

$$
\begin{aligned}
& \mathbb{E}\left(W^{1-k-2 m} H^{k}\right) \\
= & \left.(-1)^{k+m} 2^{k+3 m-1 / 2} \pi^{1 / 2} \frac{m !}{(2 m) !} \mathrm{D}_{x}^{2 m} \mathrm{D}_{y}^{k}\left(\frac{e^{x}}{F(x, y)^{2}}\right)\right|_{x=y=0}
\end{aligned}
$$




$$
\begin{aligned}
& =\left.(-1)^{k+m} 2^{k+3 m-1 / 2} \pi^{1 / 2} \frac{m !}{(2 m) !} \mathrm{D}_{x}^{2 m}\left(e^{x} \psi_{k}(x)\right)\right|_{x=0} \\
& =(-1)^{k+m} 2^{k+3 m-1 / 2} \pi^{1 / 2} \frac{m !}{(2 m) !} \sum_{j=0}^{2 m}\left(\begin{array}{c}
2 m \\
j
\end{array}\right) \mathrm{D}^{j} \psi_{k}(0) .
\end{aligned}
$$

Next, write

$$
g(y):=F(0, y)=\sum_{n=0}^{\infty} \frac{y^{n}}{n !(n+1) !} .
$$

This is the hypergeometric function ${ }_{0} F_{1}(; 2 ; y)$ and can be expressed in the modified Bessel function $I_{1}$ by $g(y)=y^{-1 / 2} I_{1}(2 \sqrt{y})[14,(5.78)]$. The special case $m=0$ of Theorem 2.8 gives the following formula.

Corollary 2.9. For any integer $k \geqq 0$,

$\mathbb{E}\left(W^{1-k} H^{k}\right)=(-1)^{k} 2^{k-1 / 2} \pi^{1 / 2} \psi_{k}(0)=\left.(-1)^{k} 2^{k-1 / 2} \pi^{1 / 2} \mathrm{D}_{y}^{k}\left(g(y)^{-2}\right)\right|_{y=0}$.

We can also obtain results when $s+t=1,-1,-3, \ldots$ as in Theorem 2.8 but $s$ and $t$ not necessarily are integers. For simplicity we treat only the case $s+t=1$, as in Corollary 2.9; this is a limiting case of Theorem 2.1.

Theorem 2.10. For any $t$ and any integer $m \geqq 0$ with $m>\operatorname{Re}(t)$,

$$
\mathbb{E}\left(W^{1-t} H^{t}\right)=(-1)^{m} \frac{2^{t-1 / 2} \pi^{1 / 2}}{\Gamma(m-t)} \int_{0}^{\infty} y^{m-1-t} \mathrm{D}_{y}^{m}\left(g(y)^{-2}\right) d y .
$$

\section{Examples}

Example 3.1. By Theorem 2.5 and straightforward manipulations,

$$
\begin{aligned}
\mathbb{E} W H & =\sum_{n=0}^{\infty} H_{n}(n+2)(\zeta(n+1)-\zeta(n+2)) \\
& =\sum_{m=2}^{\infty}\left(H_{m-1}+\frac{m}{m-1}\right)(\zeta(m)-1) \\
& =\sum_{n=1}^{\infty} \sum_{k=2}^{\infty}\left(H_{n}+1+\frac{1}{n}\right) k^{-n-1}
\end{aligned}
$$




$$
\begin{aligned}
& =\sum_{k=2}^{\infty}\left(\sum_{j=1}^{\infty} \frac{1}{j} \sum_{n=j}^{\infty} k^{-n-1}+\frac{1}{k(k-1)}-\frac{1}{k} \ln (1-1 / k)\right) \\
& =1+\sum_{k=2}^{\infty} \frac{2 k-1}{k(k-1)} \ln \frac{k}{k-1} .
\end{aligned}
$$

We do not know any closed form of this, but a numerical summation (by Maple) of one of these formulas yields

$$
\mathbb{E}(W H) \doteq 3.0462774529 \text {. }
$$

Since, by (1.6) and (1.5), $\mathbb{E} W=\sqrt{\pi / 2}$ and $\mathbb{E} H=\sqrt{2 \pi}$,

$$
\operatorname{Cov}(W, H)=\mathbb{E} W H-\pi \doteq-0.0953152007 .
$$

Thus the covariance is, as expected, negative. We have, also by (1.6), $\operatorname{Var}(W)=\pi(\pi-3) / 6$, which leads to the correlation coefficient

$$
\operatorname{Corr}(W, H) \doteq-0.6428251027 .
$$

Example 3.2. Similarly, Theorem 2.5 and Maple yield

$$
\mathbb{E}\left(W^{2} H\right) \doteq 3.8791921108 .
$$

Example 3.3. Theorem 2.6 and Maple yield (now with a little more effort to control rounding errors)

$$
\begin{aligned}
\mathbb{E}\left(W H^{2}\right) & \doteq 7.7549475561, \\
\mathbb{E}\left(W^{2} H^{2}\right) & \doteq 9.5811170671
\end{aligned}
$$

Example 3.4. Theorem 2.8 yields, for example,

$$
\begin{aligned}
\mathbb{E}\left(W^{-2} H\right) & =\frac{7}{9} \sqrt{2 \pi}, & \mathbb{E}\left(W^{-4} H\right) & =\frac{488}{675} \sqrt{2 \pi}, \\
\mathbb{E}\left(W^{-1} H^{2}\right) & =\frac{7}{3} \sqrt{2 \pi}, & \mathbb{E}\left(W^{-3} H^{2}\right) & =\frac{19}{9} \sqrt{2 \pi} .
\end{aligned}
$$

ExAmple 3.5. Theorem 2.10 with $t=-1, m=0$, the change of variable $y=(z / 2)^{2}$ and a numerical integration by Maple yield

$$
\mathbb{E}\left(W^{2} H^{-1}\right)=\frac{\sqrt{2 \pi}}{4} \int_{0}^{\infty} g(y)^{-2} d y=\frac{\sqrt{2 \pi}}{32} \int_{0}^{\infty} z^{3} I_{1}(z)^{-2} d z \doteq 0.7301458555 .
$$




\section{Proofs}

By (2.1) and (2.3) we obtain, for $\operatorname{Re}(t)<0$ and $\operatorname{Re}(s+t)>1$, using the substitutions $x=2 \lambda$ and $y=\alpha^{2}$,

$$
\begin{aligned}
\mathbb{E}\left(W^{s} H^{t}\right) & =\frac{\pi^{1 / 2} 2^{2+t / 2-s / 2}}{\Gamma(-t) \Gamma\left(\frac{s+t-1}{2}\right)} \int_{0}^{\infty} \int_{0}^{\infty} \frac{\lambda^{s+t-2} \alpha^{-1-2 t} e^{2 \lambda} d \alpha d \lambda}{F^{2}\left(2 \lambda, \alpha^{2}\right)} \\
& =\frac{\pi^{1 / 2} 2^{2-t / 2-3 s / 2}}{\Gamma(-t) \Gamma\left(\frac{s+t-1}{2}\right)} \int_{0}^{\infty} \int_{0}^{\infty} \frac{x^{s+t-2} y^{-1-t} e^{x} d x d y}{F^{2}(x, y)}
\end{aligned}
$$

This is the case $m=0$ of Theorem 2.1.

Before proceeding, we give some simple (and rather crude) estimates that, among other things, show that the integrals above converge.

Lemma 4.1. For some $c>0$ and all $x, y \geqq 0$,

(i) $F(x, y) \geqq F(x, 0)=f_{0}(x)=\sum_{n=0}^{\infty} \frac{x^{n}}{(n+1) !}=\frac{e^{x}-1}{x} \geqq c \frac{e^{x}}{1+x}$;

(ii) $F(x, y) \geqq F(0, y)=g(y)=\sum_{n=0}^{\infty} \frac{y^{n}}{n !(n+1) !} \geqq c \frac{e^{2 \sqrt{y}}}{1+y}$;

(iii) for every $m \geqq 0, \mathrm{D}_{y}^{m} F(x, y) \leqq F(x, y)$.

Proof. (i): immediate.

(ii): It remains only to check the final inequality. This is clear for $0 \leqq$ $y \leqq 1$, provided $c$ is sufficiently small. For $y>1$, take $n:=\lfloor\sqrt{y}\rfloor$ and obtain (using Stirling's formula),

$$
F(0, y) \geqq \frac{y^{n}}{n !(n+1) !} \geqq \frac{n^{2 n}}{(n !)^{2}(n+1)} \geqq c_{1} \frac{e^{2 n}}{n(n+1)} \geqq c_{2} \frac{e^{2(n+1)}}{n^{2}} \geqq c_{2} \frac{e^{2 \sqrt{y}}}{y} .
$$

(iii): It follows from (2.2) that

$$
\begin{aligned}
\mathrm{D}_{y}^{m} F(x, y) & \leqq \sum_{n=m}^{\infty} \frac{n !}{(n-m) !} \cdot \frac{((m+1) x+y) \cdots(n x+y)}{n !(n+1) !} \\
& =\sum_{j=0}^{\infty} \frac{((m+1) x+y) \cdots((m+j) x+y)}{j !(j+m+1) !} \leqq F(x, y)
\end{aligned}
$$


because $\frac{(m+i) x+y}{m+i} \leqq \frac{i x+y}{i}$.

Lemma 4.2. For every $m \geqq 0$ there are constants $C_{m}$ and $C_{m}^{\prime}$ such that, for all $x, y \geqq 0$,

$$
\left|\mathrm{D}_{y}^{m}\left(F(x, y)^{-2}\right)\right| \leqq C_{m} F(x, y)^{-2} \leqq C_{m}^{\prime}(1+x)^{2}(1+y) e^{-3 x / 2-\sqrt{y}} .
$$

Proof. $\mathrm{D}_{y}^{m}\left(F(x, y)^{-2}\right)$ is, by induction, a linear combination of terms of the type $\mathrm{D}_{y}^{k_{1}} F \cdots \mathrm{D}_{y}^{k_{l}} F / F^{l+2}$, with $l \geqq 0$ and $k_{1}, \ldots, k_{l} \geqq 1$. By Lemma 4.1(iii), each such term is bounded by $F^{-2}$, and the first inequality follows.

For the second inequality we use Lemma 4.1(i)(ii), which yield

$$
F(x, y)^{2}=F(x, y)^{3 / 2} F(x, y)^{1 / 2} \geqq c^{2}(1+x)^{-3 / 2}(1+y)^{-1 / 2} e^{3 x / 2} e^{\sqrt{y}} .
$$

Proof of Theorem 2.1. It follows from Lemma 4.2 that the double integral in (2.4) converges absolutely in the region $\operatorname{Re}(s+t)>1, \operatorname{Re}(t)<m$, uniformly on compact sets; the integral thus defines an analytic function in this region.

Next, assume $\operatorname{Re}(s+t)>1$ and $\operatorname{Re}(t)<0$. When $m=0$, the formula (2.4) is (4.1) which, as shown above, follows directly from Donati-Martin [10]. Now assume that (2.4) holds for some $m$. For each $x>0$, by integration by parts,

$$
\begin{aligned}
& \frac{1}{\Gamma(m-t)} \int_{0}^{\infty} y^{m-1-t} \mathrm{D}_{y}^{m}\left(F(x, y)^{-2}\right) d y \\
= & {\left[\frac{y^{m-t}}{\Gamma(m+1-t)} \mathrm{D}_{y}^{m}\left(F(x, y)^{-2}\right)\right]_{y=0}^{\infty} } \\
& -\frac{1}{\Gamma(m+1-t)} \int_{0}^{\infty} y^{m-t} \mathrm{D}_{y}^{m+1}\left(F(x, y)^{-2}\right) d y,
\end{aligned}
$$

where the integrated term vanishes by Lemma 4.2. Multiplying by $x^{s+t-2} e^{x}$ and integrating, we see that (2.4) holds for $m+1$ too.

By induction, we have thus shown that (2.4) holds for any $m \geqq 0$ when $\operatorname{Re}(s+t)>1$ and $\operatorname{Re}(t)<0$. Since both sides of $(2.4)$ are analytic in the domain $\operatorname{Re}(s+t)>1$ and $\operatorname{Re}(t)<m,(2.4)$ holds in this domain by analytic continuation. 
Proof of Theorem 2.2. We apply Theorem 2.1 with $t=k$ and $m=$ $k+1$ and obtain

$$
\begin{aligned}
& \mathbb{E}\left(W^{s} H^{k}\right) \\
= & (-1)^{k+1} \frac{2^{2-k / 2-3 s / 2} \pi^{1 / 2}}{\Gamma\left(\frac{s+k-1}{2}\right)} \int_{0}^{\infty} x^{s+k-2} e^{x} \int_{0}^{\infty} \mathrm{D}_{y}^{k+1}\left(F(x, y)^{-2}\right) d y d x .
\end{aligned}
$$

The inner integral equals, using Lemma 4.2 again, $-\left.\mathrm{D}_{y}^{k}\left(F(x, y)^{-2}\right)\right|_{y=0}$.

As remarked above, the functions $\psi_{k}:=\left.\mathrm{D}_{y}^{k}\left(F(x, y)^{-2}\right)\right|_{y=0}$ can be expressed in $f_{0}, f_{1}, \ldots, f_{k}$ defined in (2.6) by successive differentiations, for example, with $f=f_{0}$,

$$
\begin{aligned}
& \psi_{1}(x)=\left.\mathrm{D}_{y}\left(F(x, y)^{-2}\right)\right|_{y=0}=-2 \frac{f_{1}(x)}{f(x)^{3}}, \\
& \psi_{2}(x)=-2 \frac{f_{2}(x)}{f(x)^{3}}+6 \frac{f_{1}(x)^{2}}{f(x)^{4}} .
\end{aligned}
$$

Corollaries 2.3 and 2.4 follow from Theorem 2.2 and (4.2), (4.3), recalling that $f(x)=f_{0}(x)=F(x, 0)=\left(e^{x}-1\right) / x$.

From the definitions (2.2) and (2.6) follow

$$
\begin{aligned}
f_{1}(x) & =\sum_{n=0}^{\infty} \frac{1}{n !(n+1) !}\left(\sum_{j=1}^{n} \frac{1}{j x}\right) \prod_{i=1}^{n}(i x)=\sum_{n=1}^{\infty} \frac{x^{n-1}}{(n+1) !} \sum_{j=1}^{n} \frac{1}{j} \\
& =\sum_{n=0}^{\infty} \frac{1}{(n+2) !} \sum_{j=1}^{n+1} \frac{1}{j} x^{n}
\end{aligned}
$$

and thus its Taylor coefficients are given by

$$
\left[x^{n}\right] f_{1}(x)=\frac{1}{(n+2) !} \sum_{j=1}^{n+1} \frac{1}{j}=\frac{H_{n+1}}{(n+2) !} .
$$

Similarly,

$$
\left[x^{n}\right] f_{2}(x)=\frac{2}{(n+3) !} \sum_{1 \leqq i<j \leqq n+2} \frac{1}{i j}=\frac{1}{(n+3) !}\left(H_{n+2}^{2}-H_{n+2}^{(2)}\right),
$$


and, in general,

$$
\left[x^{n}\right] f_{k}(x)=\frac{k !}{(n+k+1) !} \sum_{1 \leqq i_{1}<i_{2}<\cdots<i_{k} \leqq n+k} \frac{1}{i_{1} i_{2} \ldots i_{k}} .
$$

Proof of Theorems 2.5 And 2.6. Since $\zeta(z)=1+2^{-z}+O\left(3^{-\operatorname{Re}(z)}\right)$ as $\operatorname{Re}(z) \rightarrow+\infty$, it is easily seen that the sums converge geometrically, and define meromorphic functions of $s$. For Theorem 2.5, a pole must be a pole of some $(n+s+1)$ !, $\zeta(n+s)$ or $\zeta(n+s+1)$ with $n \geqq 1$, thus $s=0,-1, \ldots$; similarly there are no poles except $-1,-2, \ldots$ for Theorem 2.6. By analytic continuation, it thus suffices to prove the theorems for $s>0$, and then they follow by straightforward calculations from Corollaries 2.3 and 2.4, (4.4), (4.5), and the following lemma (which can be seen as a Mellin transform calculation, cf. [22, Section 9.1]).

Lemma 4.3. If $m \geqq 2$ is an integer and $\operatorname{Re}(s)>m-1$, then

$$
\int_{0}^{\infty} x^{s} \frac{e^{x}}{\left(e^{x}-1\right)^{m}} d x=s ! \sum_{j=1}^{\infty}\left(\begin{array}{c}
j \\
m-1
\end{array}\right) j^{-s-1}
$$

In particular,

$$
\begin{array}{ll}
\int_{0}^{\infty} x^{s} \frac{e^{x}}{\left(e^{x}-1\right)^{2}} d x=s ! \zeta(s), & \operatorname{Re}(s)>1 ; \\
\int_{0}^{\infty} x^{s} \frac{e^{x}}{\left(e^{x}-1\right)^{3}} d x=\frac{1}{2} s !(\zeta(s-1)-\zeta(s)), & \operatorname{Re}(s)>2 ; \\
\int_{0}^{\infty} x^{s} \frac{e^{x}}{\left(e^{x}-1\right)^{4}} d x=\frac{1}{6} s !(\zeta(s-2)-3 \zeta(s-1)+2 \zeta(s)), & \operatorname{Re}(s)>3 .
\end{array}
$$

Proof.

$$
\frac{e^{x}}{\left(e^{x}-1\right)^{m}}=e^{-(m-1) x}\left(1-e^{-x}\right)^{-m}=\sum_{j=m-1}^{\infty}\left(\begin{array}{c}
j \\
m-1
\end{array}\right) e^{-j x}
$$


and thus

$$
\begin{aligned}
\int_{0}^{\infty} x^{s} \frac{e^{x}}{\left(e^{x}-1\right)^{m}} d x & =\sum_{j=1}^{\infty}\left(\begin{array}{c}
j \\
m-1
\end{array}\right) \int_{0}^{\infty} x^{s} e^{-j x} d x \\
& =\sum_{j=1}^{\infty}\left(\begin{array}{c}
j \\
m-1
\end{array}\right) j^{-s-1} \Gamma(s+1) .
\end{aligned}
$$

The special cases follow easily.

Proof of Theorem 2.7. We use Theorem 2.5 with $s=-2 m+\varepsilon$ and let $\varepsilon \searrow 0$. Then

$$
\Gamma(s / 2)=\Gamma(-m+\varepsilon / 2) \sim \frac{(-1)^{m}}{m !} \Gamma(\varepsilon / 2) \sim \frac{2}{\varepsilon} \frac{(-1)^{m}}{m !}
$$

and thus

$$
\frac{1}{\Gamma(s / 2)}=\Gamma(-m+\varepsilon / 2)^{-1} \sim(-1)^{m} \frac{m !}{2} \varepsilon .
$$

Hence, only the terms in the sum with a pole at $s=-2 m$ will give a contribution as $\varepsilon \rightarrow 0$.

The possible poles are:

(i) $(n+s+1) !=\Gamma(n+s+2)$ has a simple pole at $-2 m$ if $n-2 m+2 \leqq 0$, i.e. if $n \leqq 2 m-2$. The residue of $(n+s+1)$ ! then is $(-1)^{2 m-2-n} /(2 m-$ $2-n) !$.

(ii) $\zeta(n+s)$ has a simple pole at $-2 m$ if $n-2 m=1$, i.e. if $n=2 m+1$. The residue is 1 .

(iii) $\zeta(n+s+1)$ has a simple pole at $-2 m$ if $n=2 m$. The residue is 1 .

Collecting these terms yields the result.

We need yet another lemma on Mellin transforms, which extends Lemma 4.5 under a stronger hypothesis.

Lemma 4.4. Suppose that $\phi$ is an infinitely differentiable function on $[0, \infty)$ such that $x^{N} \phi(x)$ is integrable for any $N>0$. Then the integral $\Phi(s)=\int_{0}^{\infty} x^{s-1} \phi(x) d x$ converges for $\operatorname{Re}(s)>0$ and defines an analytic function there, and $\Phi$ can be extended to a meromorphic function in the complex plane, with poles only at (possibly) $0,-1,-2, \ldots$, all poles simple, and the residues

$$
\operatorname{Res}_{s=-n} \Phi=\frac{1}{n !} \mathrm{D}^{n} \phi(0) .
$$


Proof. Fix a positive integer $N$ and use the Taylor expansion

$$
\phi(x)=\sum_{n=0}^{N} \frac{1}{n !} \mathrm{D}^{n} \phi(0) x^{n}+R_{N}(x),
$$

with $R_{N}(x)=O\left(x^{N+1}\right)$ for $0 \leqq x \leqq 1$. Thus, for $\operatorname{Re}(s)>0$,

$$
\begin{aligned}
\Phi(s) & =\int_{0}^{1} x^{s-1} \sum_{n=0}^{N} \frac{1}{n !} \mathrm{D}^{n} \phi(0) x^{n} d x+\int_{0}^{1} x^{s-1} R_{N}(x) d x+\int_{1}^{\infty} x^{s-1} \phi(x) d x \\
& =\sum_{n=0}^{N} \frac{1}{n !} \mathrm{D}^{n} \phi(0) \frac{1}{s+n}+\int_{0}^{1} x^{s-1} R_{N}(x) d x+\int_{1}^{\infty} x^{s-1} \phi(x) d x .
\end{aligned}
$$

The last two integrals define analytic functions of $s$ for $\operatorname{Re}(s)>-N-1$, so we have found a meromorphic extension of $\Phi$ to $\operatorname{Re}(s)>-N-1$, with only simple poles having the prescribed residues.

Proof of Theorem 2.8. We use Theorem 2.2, which we write as

$$
\mathbb{E}\left(W^{s} H^{k}\right)=(-1)^{k} 2^{2-k / 2-3 s / 2} \pi^{1 / 2} \Gamma\left(\frac{s+k-1}{2}\right)^{-1} I(s), \quad \operatorname{Re}(s)>1-k .
$$

By Lemma 4.4 (and Lemma 4.2), $I(s)$ can be extended to a meromorphic function in the complex plane, with poles only at $0,-1,-2, \ldots$ Since the other factors in (4.8) are entire functions, the formula (4.8) is valid for all $s \neq 0,-1,-2, \ldots$ We let $s=1-k-2 m+\varepsilon$, and let $\varepsilon \searrow 0$. Then, by Lemma 4.4,

$$
\begin{aligned}
& I(s) \sim \frac{1}{\varepsilon} \operatorname{Res}_{s=1-k-2 m}(I(s))=\frac{1}{\varepsilon} \operatorname{Res}_{s+k-1=-2 m}(I(s)) \\
& =\left.\frac{1}{\varepsilon} \frac{1}{(2 m) !} \mathrm{D}_{x}^{2 m}\left(e^{x} \psi_{k}(x)\right)\right|_{x=0}=\left.\frac{1}{\varepsilon} \frac{1}{(2 m) !} \mathrm{D}_{x}^{2 m} \mathrm{D}_{y}^{k}\left(e^{x} F(x, y)^{-2}\right)\right|_{x=y=0} .
\end{aligned}
$$

Moreover, see (4.7),

$$
\Gamma\left(\frac{s+k-1}{2}\right)^{-1}=\Gamma(-m+\varepsilon / 2)^{-1} \sim(-1)^{m} \frac{m !}{2} \varepsilon .
$$

The result follows by multiplying the factors in (4.8). 
Proof of Corollary 2.9. Immediate from Theorem 2.8. (It also follows by taking $m=k+1$ in Theorem 2.10 and integrating the derivative, or by imitating the proof of Theorem 2.10 but using Theorem 2.2.) forms.

For the non-integer case we use another simple lemma on Mellin trans-

Lemma 4.5. If $\phi$ is a continuous integrable function on $[0, \infty)$, then

$$
\varepsilon \int_{0}^{\infty} x^{\varepsilon-1} \phi(x) d x \rightarrow \phi(0), \quad \text { as } \varepsilon \searrow 0
$$

Proof. For $0<\varepsilon<1$, we have $\left|\varepsilon \int_{1}^{\infty} x^{\varepsilon-1} \phi(x) d x\right| \leqq \varepsilon \int_{1}^{\infty}|\phi(x)| d x$. Moreover, letting $y=x^{\varepsilon}$,

$$
\varepsilon \int_{0}^{1} x^{\varepsilon-1} \phi(x) d x=\varepsilon \int_{0}^{1} \phi\left(y^{1 / \varepsilon}\right) d y \rightarrow \phi(0), \quad \text { as } \varepsilon \searrow 0,
$$

by dominated (bounded) convergence.

Proof of Theorem 2.10. Apply Theorem 2.1 with $s=1-t+\varepsilon$, where $\varepsilon>0$, and let $\varepsilon \searrow 0$; note that

$$
\frac{1}{\Gamma((s+t-1) / 2)}=\frac{1}{\Gamma(\varepsilon / 2)} \sim \frac{\varepsilon}{2}
$$

and use Lemma 4.5 with

$$
\phi(x)=e^{x} \int_{0}^{\infty} y^{m-1-t} \mathrm{D}_{y}^{m}\left(F(x, y)^{-2}\right) d y .
$$

(Continuity and integrability of $\phi$ follows from Lemma 4.2.)

Proof of Theorem 1.1. We have to show that for at least one pair $(s, t), \mathbb{E}\left((2 W)^{s} H^{t}\right) \neq \mathbb{E}\left(H^{s}(2 W)^{t}\right)$. We can for example take the pair $(2,1)$. By Examples 3.2 and 3.3,

$$
\begin{aligned}
& \mathbb{E}\left((2 W)^{2} H\right) \doteq 15.516768, \\
& \mathbb{E}\left((2 W) H^{2}\right) \doteq 15.509895
\end{aligned}
$$


Note, however, that the relative difference is only about $1 / 2000$, so rather careful numerical estimates are required.

Another possibility is to take $s=2, t=-1$. By Examples 3.4 and 3.5,

$$
\begin{aligned}
& \mathbb{E}\left((2 W)^{-1} H^{2}\right)=\frac{7}{6} \sqrt{2 \pi} \doteq 2.92439965 \\
& \mathbb{E}\left((2 W)^{2} H^{-1}\right) \doteq 2.92058342 .
\end{aligned}
$$

The relative difference is about 0.001 , if we trust the numerical integration.

\section{REFERENCES}

[1] Aldous, D., The continuum random tree II: an overview, Stochastic Analysis (Proc., Durham, 1990), 23-70, London Math. Soc. Lecture Note Ser. 167, Cambridge Univ. Press, Cambridge, 1991. MR 93f:60010

[2] Aldous, D., The continuum random tree III, Ann. Probab., 21:1 (1993), 248-289. $M R$ 94c:60015

[3] Aldous, D., Brownian excursion conditioned on its local time, Electron. Comm. Probab., 3 (1998), 79-90. MR 99m:60115

[4] Biane, P., Pitman, J. and Yor, M., Probability laws related to the Jacobi theta and Riemann zeta functions, and Brownian excursions, Bull. Amer. Math. Soc. (N.S.), 38:4 (2001), 435-465. MR 2003b:11083

[5] Biane, P. and Yor, M., Valeurs principales associées aux temps locaux browniens, Bull. Sci. Math. (2), 111:1 (1987), 23-101. MR 88g:60188

[6] Chassaing, P. and Marckert, J.-F., Parking functions, empirical processes, and the width of rooted labeled trees, Electron. J. Combin., 8:1 (2001), Research Paper 14, 19 pp. MR 2002b:05037

[7] Chassaing, P., Marckert, J.-F. and Yor, M., The height and width of simple trees, Mathematics and Computer Science (Versailles, 2000) 17-30, Trends Math., Birkhäuser, Basel, 2000. MR 2001j:68095

[8] Chung, K. L., Excursions in Brownian motion, Ark. Mat., 14:2 (1976), 155-177. MR 57\#7791

[9] Devroye, L., Branching processes and their applications in the analysis of tree structures and tree algorithms, Probabilistic methods for algorithmic discrete mathematics, 249-314, eds. M. Habib et al., Algorithms Combin. 16, SpringerVerlag, Berlin, 1998. MR 2000m:60099

[10] Donati-Martin, C., Some remarks about the identity in law for the Bessel bridge $\int_{0}^{1} \frac{d s}{r(s)} \stackrel{(\text { law })}{=} 2 \sup _{s \leqq 1} r(s)$, Studia Sci. Math. Hungar., 37:1-2 (2001), 131-144. $M R$ 2002j: 60141

[11] Drmota, M. and Gittenberger, B., On the profile of random trees, Random Struct. Alg., 10:4 (1997), 421-451. MR 99c:05176

[12] Drmota, M., and Gittenberger, B., The width of Galton-Watson trees conditioned by the size, Discr. Math. Theor. Comput. Sci., 6:2 (2004), 387-400. $M R$ 2005f: 60180

[13] Flajolet, P., Gao, Z., Odlyzko, A. and Richmond, B., The distribution of heights of binary trees and other simple trees, Combin. Probab. Comput., 2 (1993), 145-156. MR 94k:05061 
[14] Graham, R. L., Knuth, D. E. and Patashnik, O., Concrete Mathematics, 2nd ed., Addison-Wesley, Reading, Mass (1994). MR 97d:68003

[15] Janson, S., Random cutting and records in deterministic and random trees, Random Struct. Alg., 29:2 (2006), 139-179. MR 2007k:05200

[16] Jeulin, T., Semi-martingales et grossissement d'une filtration, Lecture Notes in Mathematics 833, Springer-Verlag, Berlin, 1980. MR 82 h:60106

[17] Kennedy, D. P., The distribution of the maximum Brownian excursion, J. Appl. Probab., 13:2 (1976), 371-376. MR 53\#6769

[18] Louchard, G., Kac's formula, Lévy's local time and Brownian excursion, J. Appl. Probab., 21:3 (1984), 479-499. MR 86f:60100

[19] Meir, A. and Moon, J. W., On the altitude of nodes in random trees, Canad. J. Math., 30 (1978), 997-1015. MR 80k:05043

[20] Pitman, J. and Yor, M., Decomposition at the maximum for excursions and bridges of one-dimensional diffusions, Itô's stochastic calculus and probability theory, 293-310, Springer, Tokyo, 1996. MR 98f:60153

[21] Rényi, A. and Szeneres, G., On the height of trees, J. Austral. Math. Soc., 7 (1967), 497-507. MR 36\#2522

[22] Szpankowski, W., Average Case Analysis of Algorithms on Sequences, Wiley, New York, 2001. $M R$ 2002f: 68001

[23] TAKÁCS, L., Limit distributions for queues and random rooted trees, J. Appl. Math. Stochastic Anal., 6:3 (1993), 189-216. MR 94m:60194

[24] Yor, M., Some aspects of Brownian motion. Part II: Some recent martingale problems. Lectures in Mathematics ETH Zürich, Birkhäuser Verlag, Basel, 1997. $M R$ 98e: 60140 